\title{
Influence of Coexisting Functionalized Polyalkylmethacrylates on the Formation of ZnDTP-Derived Tribofilm
}

\begin{abstract}
Although zinc dialkyldithiophosphate (ZnDTP) shows supreme antiwear performance by forming a thick polyphosphate-based tribofilm on the friction surface, it has been pointed out that the use of $\mathrm{ZnDTP}$ is accompanied by interference from the coexisting dispersant. For the maintenance of the good antiwear performance of $\mathrm{ZnDTP}$ when it is used with coexisting polar compounds, the effects of polar compounds on the tribofilm formation of ZnDTP should be clarified. Since the recent demand for minimizing energy consumption recommends applying lower-viscosity oil to reduce the viscous drag, the use of a good viscosity modifier (VM) that improves the temperature-viscosity characteristic of the oil is required. Here we tested several types of functionalized polymethacrylate (PMA)-VMs used with ZnDTP, and we discuss their effect on the tribofilm formation of ZnDTP. We observed that the PMA-series VMs with different functional groups influenced the tribofilm formation of ZnDTP. The amino-group-containing PMA in particular significantly reduced the formation of tribofilm.
\end{abstract}

\section{Keywords}

Lubricant additives, Tribochemistry, Surface chemical analysis, Boundary lubrication 


\section{Introduction}

The most important tribological issue in the automotive industry at this time is to minimize the engine friction torque to attain optimal fuel efficiency. Based on the International Council on Clean Transportation (ICCT)'s 2014 report [1], the fuel efficiency requirement expressed as the $\mathrm{CO}_{2}$ emission will become $<100 \mathrm{~g} / \mathrm{km}$ in $2020-2025$. The reduction of engine oil viscosity is one of the most effective key methods that could be used to reach this fuel efficiency requirement. Engine oil viscosity has been regulated for many years for many reasons, and the agreed viscosity grades are listed in the SAE J300 standard issued by the Society of Automotive Engineers (SAE).

Low-viscosity oils are generally classified by their high-temperature high-shear-rate (HTHS) viscosities. In April 2013, the engine oil viscosity classification standard added SAE16 (> $2.3 \mathrm{mPa}$ s HTHS) as the lowest high-temperature viscosity grade [2]. However, SAE 20 (> $2.6 \mathrm{mPa}$ s HTHS), which is the former lowest-viscosity grade oil, is still used as a low-viscosity engine oil to avoid engine trouble. In January 2015, SAE J300 was revised by the addition of two new high-temperature viscosity grades: SAE 12 and SAE 8 (> 2.0 and 1.7 $\mathrm{mPa}$ s HTHS, respectively) [3]. Although the current lowest-viscosity grade in the SAE specifications is SAE 8, which is not yet used commonly for commercial engine oil, the use of these low-viscosity engine oils is inevitable in the near future to attain ultimate fuel economy. In addition, the role of viscosity modifiers (VMs), which are also known as viscosity index improvers (VIIs), becomes more important to the fuel economy because they can be used to depress the viscosity at low-temperature regions more effectively.

However, since the use of low-viscosity oil results in an increase in operations in the boundary lubrication regime, wear and scuffing problems become a major concern. The action of the antiwear agent thus becomes more important. In the case of engine oil, zinc dialkyldithiophosphate (ZnDTP; ZDDP is also often used as acronym of this compound) has 
been used as an antiwear agent for many years, and almost no alternative additive is used, with a few exceptions [4].

ZnDTP has shown superior antiwear performance and anti-scuffing performance, which are collectively referred to as the "load-carrying capability," by forming a phosphorous-containing tribofilm on the surface. However, since the phosphorus in exhaust gas poisons the exhaust catalysts, the phosphorous concentration in engine oil has been decreased in accord with regulations. With the reduction of both the viscosity and the ZnDTP concentration, it becomes very difficult to create new engine oils that maintain good load-carrying capability.

There have been a great number of studies regarding the lubrication performance of ZnDTP-formulated lubricants and their tribofilm formation that included precise analyses of the nature or chemical composition of the ZnDTP-derived tribofilms. The earlier studies of the ZnDTP tribofilms focused on the thermal decomposition and characterization of deposited debris [5-8]. With the advances in equipment and surface analytical tools, a direct characterization of tribofilms produced on the surface by rubbing in the ZnDTP-containing oil has been carried out, clarifying the structure of the tribofilms [9-18]. Details can be found in a review paper [19], and a schematic illustration of ZnDTP-derived tribofilm that illustrates the morphology and composition is available in several papers [19-23].

Tribological research concerning ZnDTP has been conducted by many researchers. Some of the recent topics are (1) the synergism between ZnDTP and molybdenum dialkyldithiocarbamate (MoDTC) that works to reduce boundary friction more efficiently, and (2) the performance of $\mathrm{ZnDTP}$ on non-ferrous materials or coatings such as amorphous carbon-coated surfaces [24-27].

Tribologists who are familiar with additive performances understand that a ZnDTP-derived tribofilm is composed of a mass of polyphosphate glass [ also sometimes 
referred to as poly(thio)phosphate] with a higher degree of polymerization at the outermost surface and a lower degree of polymerization underneath together with sulfide and oxide of zinc and iron, which produces a relatively rougher surface morphology.

Since commercial lubricating oils generally contain several types of additives such as oxidation inhibitors, load-carrying additives, friction modifiers, etc., a given additive does not always perform well compared with its single application. One of the typical examples of the antagonism between additives is between $\mathrm{ZnDTP}$ and ashless dispersant in engine oils. A reduction of the load-carrying capability of ZnDTP by the ashless dispersant and the reaction mechanism have been proposed $[18,28,29]$.

Despite the complexity of the additive formulation for commercial lubricating oils, the antagonism and synergistic actions between additives has attracted researchers' interest [30-32]. However, these actions have not yet been defined in explicit detail. An understanding of the effects of coexisting polar compounds on the performance of ZnDTP is very important to attain sufficient performance with a small concentration of ZnDTP.

As noted above, the improvement of the temperature-viscosity characteristics of engine oils is essential to attain the ultimate fuel economy, and the identification of the best VM will thus be a crucial step. Among the several types of polymers that could be used for VM additives, polymethacrylate (PMA) has been known to show excellent temperature-viscosity characteristics of lubricating oils. There are two types of PMA-VM: the nondispersant type and the dispersant type. Nondispersant-type PMAs are composed of a random homopolymer of alkylmethacrylate, and dispersant-type PMAs are composed of a random copolymer of alkylmethacrylate and a functionalized-methacrylate such as dimethylaminomethacrylate. Dispersant-type PMAs were developed as multifunctional additives that have both a viscosity index improver (VII) and a dispersant.

Although the main role of the dispersant-type PMAs is the improvement of the bulk 
property, and they were found to possess a boundary lubrication ability by forming molecular layers adsorbed at the polar functional groups [33-39]. Since the improvement of the boundary lubrication performance including friction reduction is also an important issue for the use of lower-viscosity oils, the action of the adsorbed organic molecular layers becomes more important.

In these circumstances, the objective of the present study was to understand the effects of polar compounds on the tribofilm formation of ZnDTP by focusing on the usage of PMA-type viscosity modifiers in new engine oils.

\section{Experimental details}

\subsection{Sample oil}

We used poly- $\alpha$-olefin (PAO) with the viscosities of $16.89 \mathrm{~mm}^{2} / \mathrm{s}$ at $40^{\circ} \mathrm{C}$ and $3.88 \mathrm{~mm}^{2} / \mathrm{s}$ at $100^{\circ} \mathrm{C}$ formulated with zinc dioctyldithiophosphate (prim-C8 ZnDTP; the alkyl chain was 2-ethylhexyl) as a standard sample oil to generate a tribofilm on the friction surface. Although the phosphorus concentration level in the current engine oils (GF-5) is 0.08 mass $\% \mathrm{P}$, the concentration of 0.1 mass $\% \mathrm{P}$ was used in the present study to observe the recognizable tribofilm formation.

As coexisting additives, we used four types of PMA that are model VM compounds. One type was an ordinary random homopolymer of tetradecylmethacrylate, and the other three types were random copolymers of tetradecylmethacrylate plus one of three types of functionalized methacrylate: 2-hydroxyethylmethacrylate, dimethylaminomethacrylate, and methacrylic acid. We tentatively named these four PMAs 'PMA-O(zero),' 'PMA-OH,' 'PMA-N,' and 'PMA-COOH' based on the functional group used.

The mean molecular weights of the PMAs was adjusted to 20,000. The mass ratios of the monomers of the methacrylate and the functionalized methacrylate prepared for 
polymerization were 83:17 (PMA-N), 90:10 (PMA-OH), and 93:7 (PMA-COOH), which

produced similar concentrations of functionalized methacrylate as $21 \mathrm{~mol} \%$ (PMA-N), and 19 mol\% (PMA-OH and PMA-COOH).

In addition, as a model dispersant compound, we used N-tetraethylenepentamin polyisobutylene succinimide for a reference. The mean molecular weight of the polyisobutylene (PIB) moiety was 1,000. The chemical formulas of these samples are shown in Figure 1.

\subsection{Tribometer}

A ball-on-disk-type rolling-sliding tribometer was used. The ball and disk were rotated by a separate motor to provide any rotating speed needed. The ball was that for a commercially available rolling bearing, made of heat-treated high-carbon chromium bearing steel (JIS SUJ 2, equivalent to AISI 52100; composition \%: C 0.95-1.10, Si 0.15-0.35, $\mathrm{Mn}<0.50, \mathrm{P}<0.025$, $\mathrm{S}<0.025$, Cr 1.30-1.60; hardness: HRC > 60) with a dia. of $25.4 \mathrm{~mm}$. The ball was mounted on a rotating shaft with a specially designed cap nut. The disk was a commercially available thrust washer for a thrust needle roller bearing, made of carbon tool steel (JIS SK 85; composition \%: C 0.80-0.90, Si 0.10-0.35, Mn 0.10-0.50, P < 0.030, S < 0.030; hardness: HRC > 60) with the following dimensions: outer dia. $27 \mathrm{~mm}$, inner dia. $16 \mathrm{~mm}$, thickness 3 $\mathrm{mm}$. The disk specimen was mounted on another rotating shaft in an oil cup with a fixing jig. Since the surface roughness of the disk as received was approx. $R a=0.2 \mu \mathrm{m}$ (which is somewhat too rough for the surface analysis), we polished the disk surface with an electric fine-polisher and fine polishing slurry, followed by buffing to attain roughness $<R a=0.02 \mu \mathrm{m}$. The contact point of the ball and disk was immersed in the sample oil, and the experiments were conducted under fully flooded conditions under a controlled oil temperature.

As summarized in Table 1, the applied load $W$ was $63.9 \mathrm{~N}$, and the mean contact 
pressure $P_{\text {mean }}$ was $667 \mathrm{MPa}$; the entrainment speed $u$ [i.e., $\left(u_{\text {ball }}-u_{\text {disk }}\right) / 2$ ] was $80 \mathrm{~mm} / \mathrm{s}$, and

$100^{\circ} \mathrm{C}$. The tribo-experiments were conducted under so-called boundary lubrication conditions, judging from the calculated $\Lambda$ value $(=h / \sigma)$ of 0.73 .

\subsection{Surface Analysis}

\subsubsection{Optical image and roughness}

The optical images and surface roughness were measured using a 3D laser scanning confocal microscope.

\subsubsection{Auger electron spectroscopy (AES)}

We conducted an auger electron spectroscopy (AES) analysis to study the thickness and chemical composition of the ZnDTP-derived tribofilms on the friction surface. For the identification of the position of the tribofilm observed inside a wear track, we observed the scanning electron microscopic (SEM) images by changing the magnification from 100 to 5000-fold. Figure 2 shows typical SEM images (SEI: secondary electron image, and COMPO: composition image) taken after 24-hour tests with the standard sample oil containing ZnDTP.

At the 5000-fold magnification, we carried out a chemical analysis at several positions of $3 \mu \mathrm{m} \times 3 \mu \mathrm{m}$ in size. By applying alternate argon ion sputtering and the AES chemical analysis, we obtained the depth profiles of the chemical elements at the targeted area. Each argon ion sputtering per scan applied in this study was equivalent to the sputtering depth of approx. $4 \mathrm{~nm}$ per scan in the case of $\mathrm{SiO}_{2}$ surface.

As has been reported in many studies, ZnDTP-formulated oil is known to produce a patchy island-like tribofilm on the friction surface. We also observed this phenomenon after a 
1-hr tribotest with the ZnDTP-formulated standard sample oil, as shown in Figure 3. We therefore selected three different positions that might have different thicknesses judging from the appearance of the SEI images and the brightness of the COMPO images. The three positions were tentatively named the thick, medium, and thin regions. At each position, the depth profile of the chemical elements was obtained separately.

Each spectrum obtained was processed with the AES apparatus' equipped software to determine the intensities of the chemical elements. In this study, the targeted elements were P, $\mathrm{S}, \mathrm{O}, \mathrm{Zn}$, and $\mathrm{Fe}$. After the argon ion sputtering of $>30$ or 50 cycles depending on the triboconditions, the intensities of $\mathrm{P}, \mathrm{S}, \mathrm{Zn}$, and $\mathrm{O}$ showed constant, very small noise level values. In this study, we subtracted the noise-level values obtained from the entire intensity of each element along the depth. In the case of Fe, this type of noise level intensity was obtained from the measurement of pure $\mathrm{Cu}$ under the same analytical conditions. Lastly, we converted the corrected intensities to atomic concentrations using the relative sensitivity coefficient of each chemical element.

\subsubsection{Electron probe micro-analysis (EPMA)}

Compared with AES, which can analyze thinner surfaces of $<10 \mathrm{~nm}$, an electron probe micro-analysis (EPMA) gives only the total average value of each element at the targeted area. However, an EPMA can be used to obtain the chemical analysis of a wide area along the whole width of a wear track perpendicular to the sliding direction, which was approx. $400 \mu \mathrm{m}$ in the present experiments. In this study, we used the EPMA chemical imaging data to determine the distribution of elements over the whole width of the wear track. The whole image size was $100 \mu \mathrm{m} \times 500 \mu \mathrm{m}$ and consisted of 12,500 pixels $(50 \times 250)$, in which the size of each pixel was $2 \mu \mathrm{m} \times 2 \mu \mathrm{m}$. 


\section{Results and Discussion}

\subsection{Standard sample oil formulated with ZnDTP alone}

Figure 4 shows the friction coefficient changes with the friction time. Separate tests were carried out using the friction times of 1, 4, 7, 10 and $24 \mathrm{hrs}$, and the friction coefficients of the last 5 min of each test were averaged and plotted to make Figure 4, which shows that the friction coefficient gradually decreased and settled at a steady value after 10 hrs. For every disk specimen with a rubbing time of 1, 4, 7, 10 and 24 hrs, we performed an AES depth analysis. Typical results are shown in Figure 5.

Based on all of the depth profile data of the thick and medium regions, it was apparent that the phosphorous-based tribofilm was composed of two different layers. One layer was located at the outermost surface, in which the concentration of phosphorous was almost constant toward the depth direction. We tentatively named this layer the 'deposited film.' The second layer was located underneath the deposited film, and at this layer the concentration of phosphorous gradually decreased to zero toward the depth direction. This was named the 'gradient layer.'

In the gradient layer, the concentrations of both zinc and oxygen also decreased gradually toward the depth direction of the specimen, and in response to this, the concentration of iron gradually increased. This two-layer structure of ZnDTP-derived tribofilm was already reported [21], and we might have observed the same phenomena.

Figures 6 shows the thicknesses of the ZnDTP-derived tribofilm at the three different areas regarded as thick, medium and thin regions. Figure 7 shows the chemical composition of the deposited layer, which was the averaged value obtained at the thick and medium regions. Both the thickness and the phosphorous concentration in the chemical composition of the deposited film tended to decrease gradually with time, and settled at a certain steady value after $10 \mathrm{hrs}$. This shows that ZnDTP generated $\mathrm{z}$ rather thick tribofilm at the initial 
region of the friction period, but after a certain time the thickness of the tribofilm decreased gradually and settled into a steady-state condition that was determined by the relationship between the formation and the wear of the tribofilm. A similar observation was reported by Fujita et al. [40, 41]. We selected 24 hrs of friction time as a standard condition to compare the effects of coexisting additives on the tribofilm formation of ZnDTP.

\subsection{Effect of coexisting additives on the formation of a tribofilm}

\subsubsection{AES analysis}

The thicknesses and chemical compositions of the tribofilms are compared in Figure 8a,b and Figure $9 \mathrm{a}-\mathrm{c}$. The tribofilm thicknesses observed at the thick and medium regions are shown in Figure 8a. The chemical compositions of each tribofilm that was the average of the values observed at the thick and medium regions are compared in Figure 8b. Since the thick, medium, and thin regions were selected visually from the SEM images, they did not always correspond correctly to the actual thickness order, but they seemed to correspond to the total thickness of the tribofilm (deposited film + gradient layer). In terms of deposited film, the thickness was almost the same considering the difference between the thick and medium regions. In contrast, the thickness of the gradient layer was affected by the coexisting additive.

The comparisons of the concentrations of $\mathrm{P}, \mathrm{O}, \mathrm{S}$, and $\mathrm{Zn}$ elements that were the averaged values observed at the thick and medium regions are shown individually in Figure 9a. In order to show the effects of the coexisting additives clearly, the data are plotted as the relative concentration that was obtained by dividing the value of each element by that in the case of standard oil in Figure 9b. The elemental ratios of selected elements are shown in Figure 9c.

From these figures, we observed first that whereas the $\mathrm{P} / \mathrm{Zn}$ atomic ratio in the $\mathrm{ZnDTP}$ 
molecule is 2.0, the $\mathrm{P} / \mathrm{Zn}$ ratio in the deposited film was approx. 0.45.

Considering the decomposition mechanism of $\mathrm{ZnDTP}$, if most of the zinc atom was first uncoupled and presented in the deposited film as $\mathrm{ZnO}$ or $\mathrm{ZnS}$ as inorganic deposits and the phosphorus-based deposited film was generated from the remaining organic moiety in which the exchanging reaction between sulfur and oxygen occurred simultaneously, this small value of $\mathrm{P} / \mathrm{Zn} \sim 0.45$ means that only $23 \%$ of total phosphorus was used to generate the phosphate-based deposited film, and the remaining $77 \%$ of the phosphorus was in the form of oil-soluble compounds without $\mathrm{Zn}$ in the oil [13, 42].

In addition, the elemental ratio of $\mathrm{O} / \mathrm{P}$ was maintained almost 4 , which means the deposited film was phosphate-based tribofilm that accorded with the observation by many researchers already reported.

As shown in Figure 8a,b and Figure 9a,b, the effect of the Dispersant and PMA-N (which contained amino groups) was significant among the additives. A characteristic feature of the tribofilm with both amino-containing compounds (PMA-N and Dispersant) was a low phosphorous concentration together with high sulfur and iron concentrations. The low concentration of oxygen in the tribofilm of +PMA-N and +Dispersant corresponded to the concentration of phosphorus, resulting in the similar $\mathrm{O} / \mathrm{P}$ values in all of the cases.

We observed that the PMA-COOH additive did not have a significant effect on the thickness or the composition of the tribofilm. In the cases of PMA-0 and PMA-OH, the tribofilms were similar to that created by ZnDTP alone, but the sulfur concentration was low.

\subsubsection{EPMA results}

Although AES can precisely analyze very small areas and small depths, it is very difficult to determine the macroscopic distribution of tribofilms with the same resolution over the entire friction track by our method. Even when a similar thickness or composition was obtained 
from the small-area analyses with different coexisting compounds, it was not sufficient to identify the macroscopic overall tribofilm formation. Therefore, we used an EPMA to obtain the elemental distribution over the friction area.

Figure 10 shows the chemical images shown by the EPMA together with the optical microscope images obtained by laser scanning confocal microscopy at the same position. As shown in these pictures, the distribution of elements was very different among the additives.

As mentioned in the Experimental section, each image consisted of 12,500 pixels $(50 \times 250)$. For a quantitative discussion of the results, we used the chemical images obtained at the center part, which was 5,000 pixels $(50 \times 100)$ to make the intensity histogram shown in Figure $11 \mathrm{a}-\mathrm{d}$.

Regarding phosphorous, zinc and oxygen, the maximum intensity and distribution of these elements were similar in the four cases of $\mathrm{ZnDTP}$ alone, +PMA 0, +PMA-OH, and +PMA-COOH. In contrast to these four cases, both the amino-containing additives of + Dispersant and +PMA-N showed a shift of the maximum intensity value toward lower intensity, and the distribution became sharp. This tendency was significant for phosphorous and oxygen. This result demonstrates that both amino-containing additives inhibited the formation of phosphate-based tribofilms from $\mathrm{ZnDTP}$.

In order to understand the effect more clearly and quantitatively, we identified the Mode intensity value (i.e., the maximum frequency position in intensity) from the histogram, which could be regarded as a representative concentration of chemical elements of the image. Figure 12a,b shows the results of our comparisons of the mode intensity and the relative mode intensity on the basis of $\mathrm{ZnDTP}$ alone.

These results showed that (1) the amino group-containing compounds (PMA-N and Dispersant) significantly decreased the tribofilm formation of ZnDTP (with decreases in the P, $\mathrm{O}$, and $\mathrm{Zn}$ concentrations); (2) both PMA-OH and PMA-COOH did not significantly interfere 
with the tribofilm formation, in that the thickness and composition were almost identical to those of ZnDTP alone but the sulfur content was lower; and (3) in the case of PMA-0, the tribofilm was similar to that obtained with ZnDTP alone.

Since the intensity of each chemical element of $\mathrm{P}, \mathrm{O}, \mathrm{S}$, and $\mathrm{Zn}$ was observed at the same pixel position, the correlation between the selected elements, and distribution and the location of each element can be obtained (Fig. 13a-d). A strong correlation between $\mathrm{O}$ and $\mathrm{P}$ was clearly observed, whereas the other relations were poorer. Figure $14 a, b$ shows the intensity ratio and correlation coefficients among the chemical elements. The $\mathrm{O} / \mathrm{P}$ ratio was similar in every additive, and the correlation coefficient was very high at $>0.95$.

From this observation, we can say that the distributions of $\mathrm{O}$ and $\mathrm{P}$ were similar and that the concentrations of both elements changed equally at the same location. S and $\mathrm{Zn}$ also showed a high correlation coefficient, which means that the distributions of $\mathrm{Zn}$ and S were similar and present at almost the same location. From the EPMA results, it can be also said that the amino group-containing compounds (PMA-N and Dispersant) significantly increased the S/P ratio. From both AES analyses and the EPMA data, the formation of a sulfur-rich tribofilm was confirmed. Since we did not have found this sulfur-enhancing mechanism, we will study this continuously as a future issue.

Figure $14 \mathrm{c}$ shows the relative intensity calculated with reference to ZnDTP alone. The most significant feature was the increase in sulfur content based on both the $\mathrm{S} / \mathrm{P}$ and $\mathrm{S} / \mathrm{Zn}$ relations in the cases with PMA-N and Dispersant.

\subsubsection{Comprehensive overview}

In this study, PMA-N and the Dispersant contained different types of amino groups: PMA-N had a tertiary dimethyl amino group $\left[-\mathrm{N}\left(\mathrm{CH}_{3}\right)_{2}\right]$, and the Dispersant had primary and secondary amino groups ( $>\mathrm{NH}$ and $\left.-\mathrm{NH}_{2}\right)$. Although the dimethyl amino group was expected 
to have no or less interaction with ZnDTP due to the lower reactivity and steric hindrance by the methyl groups, we observed that the effect was almost the same as the primary and secondary amino groups. Therefore, the antagonism action observed with PMA-N might be brought about by the same mechanism as in the case of the Dispersant, in which ZnDTP produced a coordination compound with an amino group in the molecule that was less likely to produce the phosphate-based tribofilm by increasing the decomposition temperature.

In the cases of the $\mathrm{OH}$ and $\mathrm{COOH}$ groups, no remarkable interaction between ZnDTP molecules in the bulk oil was observed. Although neither PMA-OH nor PMA-COOH significantly interfered with the generation of the phosphate-based tribofilm, they exerted a certain influence on the tribofilm formation that was suggested by the results of both the AES and EPMA.

\section{Conclusion}

We used both auger electron spectroscopy (AES) and an electron probe micro-analysis (EPMA) to analyze ZnDTP-derived tribofilm formation to determine the effects of coexisting polar compounds (PMAs and Dispersant). We observed that the PMA-series viscosity modifiers with different functional groups influenced the tribofilm formation of ZnDTP, although the degree of influence differed among the PMAs.

The results revealed by AES and EPMA were not always identical, but they provided some important information:

- The phosphorus-based tribofilm was composed of the two layers of a deposited film and a gradient layer underneath.

- The distribution of the tribofilm over the whole friction surface was affected by coexisting polar compounds (PMAs and Dispersant).

- The location and distribution of phosphorus and oxygen were identical for all of 


\section{Acknowledgements}

This study was supported in part by the Council for Science, Technology and Innovation (CSTI), the Cross-ministerial Strategic Innovation Promotion Program (SIP), "Innovative Combustion Technology" (Funding agency: JST) and by JX Nippon Oil \& Energy Corporation. We thank Sanyo Chemical Industries for providing the PMAs and the Center for Advanced Materials Analysis, Tokyo Institute of Technology for the assistance and discussions of the surface analytical studies.

\section{References}

[1] International Council on Clean Transportation (2014). Global passenger vehicle standards. Retrieved from http://theicct.org/info-tools/global-passenger-vehicle-standards

[2] http://standards.sae.org/j300_201304/

[3] http://standards.sae.org/j300_201501/

[4] ENEOS Premium Motor Oil SUSTINA Series. http://www.noe.jx-group.co.jp/carlife/product/oil/list/gasoline.html 
[5] Feng IM. Pyrolysis of Zinc Dialkyl Phosphorodithioate and Boundary Lubrication. Wear 1960; 3: 309-311.

[6] Dickert Jr JJ, Rowe CN. The Thermal Decomposition of Metal O,O-Dialkylphosphorodithioates. J Org Chem 1967; 32: 647-653.

[7] Brazier AD. Elliott JS. The thermal stability of zinc dithiophosphates. J. Inst. Petrol. 1967; 53: 63-76.

[8] Hanneman WW, Porter RS. The Thermal Decomposition of Dialkyl Phosphates and O,O-Dialkyl Dithiophosphates. J Org Chem 1964; 29: 2996-2998.

[9] Bird RJ. Galvin GD. The application of photoelectron spectroscopy to the study of e. p. films on lubricated surfaces. Wear 1976; 37: 143-167.

[10]Coy RC, Jones RB. The Thermal Degradation and EP Performance of Zinc Dialkyldithiophosphate Additives in White Oil. ASLE Trans. 1981; 24: 77-90.

[11] Coy RC. Jones RB. The Chemistry of the Thermal Degradation of Zinc Dialkyldithiophosphate Additives. ASLE Trans. 1981; 24: 91-97.

[12] Willermet PA, Dailey DP, Carter III RO, Schmitz PJ, Zhu W, Bell JC, Park D. The composition of lubricant-derived surface layers formed in a lubricated cam/tappet contact II. Effects of adding overbased detergent and dispersant to a simple ZDTP solution. Tribology International 1995; 28: 163-175.

[13] Willermet PA, Dailey DP, Carter III RO, Schmitz PJ, Zhu W. Mechanism of Formation of Antiwear Films from Zinc Dialkyldithiophosphates. Tribology Int. 1995; 28: 177-187.

[14]Bancroft GM. Kasrai M. Fuller M. Yin Z. Fyfe K. Tan K.H. Mechanisms of tribochemical film formation: stabilityof tribo- and thermally-generated ZDDP films. Tribology Letters 1997; 3: 47-51.

[15]Fuller M. Yin Z. Kasrai M, Bancroft GM. Yamaguchi ES. Ryason PR. Willermet PA. Tan KH. Chemical characterization of tribochemical and thermal films generated from 
neutral and basic ZDDPs using X-ray absorption spectroscopy. Tribology Int. 1997; 30: $305-315$.

[16]Fuller M. Kasrai M. Bancroft GM. Fyfe K. Tan KH. Solution decomposition of zinc dialkyl dithiophosphate and its effect on antiwear and thermal film formation studied by X-ray absorption spectroscopy. Tribology Int. 1998; 31: 627-644.

[17] Aktary M. McDermott MT. Torkelson J. Morphological evolution of films formed from thermooxidative decomposition of ZDDP. Wear 2001; 247: 172-179.

[18] Martin JM. Grossiord C. Le Mogne T. Igarashi J. Role of nitrogen in tribochemical interaction between Zndtp and succinimide in boundary lubrication. Tribology Int. 2000; 33: 453-459.

[19] Spikes H. The history and mechanisms of ZDDP. Tribology Letters, 2004; 17: 469-489.

[20] Ito K. Martin JM. Minfray C. Kato K. Formation Mechanism of a Low Friction ZDDP Tribofilm on Iron Oxide. Tribology Trans. 2007; 50: 211-216.

[21] Martin, J M. Grossiord C. Le Mogne,T. Bec,S., Tonck A. The two-layer structure of Zndtp tribofilms Part I: AES, XPS and XANES analyses. Tribology Int. 2001; 34: $523-530$.

[22]Hsu, S.M., Gates, R.S. Boundary lubricating films: formation and lubrication mechanism. Tribology Int. 2005; 38: 305-312.

[23]Bec S. Tonck A. Georges JM. Coy RC. Bell JC. Roper GW. Relationship between mechanical properties and structures of zinc dithiophosphate anti-wear films. Proc. R. Soc. Lond. A. 1999; 455: 4181-4203.

[24] Morina A. Haque T. Neville A. Comparing Tribochemical Film Formation and Durability at Steel and CrN Coating Interface in Boundary Lubrication. Tribology Online 2010; 5: 187-194.

[25] Morina A. Zhao H. Mosselmans J F W. In-situ reflection-XANES study of ZDDP and 
MoDTC lubricant films formed on steel and diamond like carbon (DLC) surfaces. Applied Surface Science 2014; 297: 167-175.

[26] Masuko M. Ono T. Aoki S. Suzuki A. Ito H. Friction and wear characteristics of DLC coatings with different hydrogen content lubricated with several Mo-containing compounds and their related compounds. Tribology Int. 2015; 82: 350-357.

[27] Tasdemir H A. Wakayama M. Tokoroyama T. Kousaka H. Umehara N. Mabuchi Y. Higuchi T. The effect of oil temperature and additive concentration on the wear of non-hydrogenated DLC coating. Tribology Int. 2014; 77: 65-71.

[28] M. Shiomi M. Tokashiki M. Tomizawa H. Kuribayashi T. Interaction between zinc dialkyldithiophosphate and amine. Lubrication Science 1989; 1: 131-147.

[29] Bartha L. Deak Gy. Kovacs M. Kocsis Z. Vuk T. Interaction of PIB-succinimides and other Engine Oil Additives. Lubrication Science 1997; 9: 173-180.

[30] Greenall A. Neville A. Morina A. Sutton M. Investigation of the interactions between a novel, organic anti-wear additive, ZDDP and over based calcium sulphonate. Tribology Int. 2012; 46: 52-61.

[31]Ratoi M. Niste V B. Alghawel H. Suen Y F. Nelson K. The impact of organic friction modifiers on engine oil tribofilms. RSC Adv. 2014; 4: 4278-4285.

[32] Sharma V. Erdemir A. Aswath P B. An analytical study of tribofilms generated by the interaction of ashless antiwear additives with ZDDP using XANES and nano-indentation. Tribology Int. 2015; 82: 43-57.

[33] Cann P M. Spikes H A. The behavior of polymer solutions in concentrated contacts: immobile surface layer formation. Tribology Trans. 1994; 37: 580-586.

[34] Smeeth M. Gunsel S. Spikes H A. Boundary film formation by viscosity index improvers. Tribology Trans.1996;39:720-5.

[35] Muller M. Topolovec-miklozic K. Dardin D. Spikes HA.The design of boundary 
film-forming PMA viscosity modifiers.TribologyTrans. 2006;49:225-32.

[36] J Fan J. Müller M. Stöhr T. Spikes HA. Reduction of friction by functionalised viscosity index improvers. Tribology letters 2007; 28: 287-298.

[37] Tohyama M. Ohmori T. Murase A. Masuko M. Friction reducing effect of multiply adsorptive organic polymer. Tribology Int. 2009; 42: 926-33.

[38] Aoki S. Yamada Y. Fukada D. Suzuki A. Masuko M. Verification of the advantages in friction-reducing performance of organic polymers having multiple adsorption sites. Tribology Int. 2013; 59: 57-66.

[39] Aoki S. Fukada D. Yamada Y. Suzuki A. Masuko M. Synergistic friction-reducing effects between the transverse direction of anisotropic surface roughness and the high-density adsorbed films under a boundary lubrication regime. Tribology Int. 2013; 58: 98-106.

[40]Fujita H. Glovnea RP. Spikes HA. Study of zinc dialkydithiophosphate antiwear film formation and removal processes, part I: Experimental - Tribology trans. 2005; 48: $558-566$.

[41]Fujita H. Spikes HA. Study of zinc dialkyldithiophosphate antiwear film formation and removal processes, part II: Kinetic model Tribology trans. 2005; 48: 567-575.

[42] Masuko M. Ohkido T. Suzuki A. Ueno T. Fundamental Study of Changes in Friction and Wear Characteristics due to ZnDTP Deterioration in Simulating Engine Oil Degradation during Use. Transient Process in Tribology, Proc. $30^{\text {th }}$ Leeds-Lyon Symposium on Tribology September 2003, 2004: 359-366. 


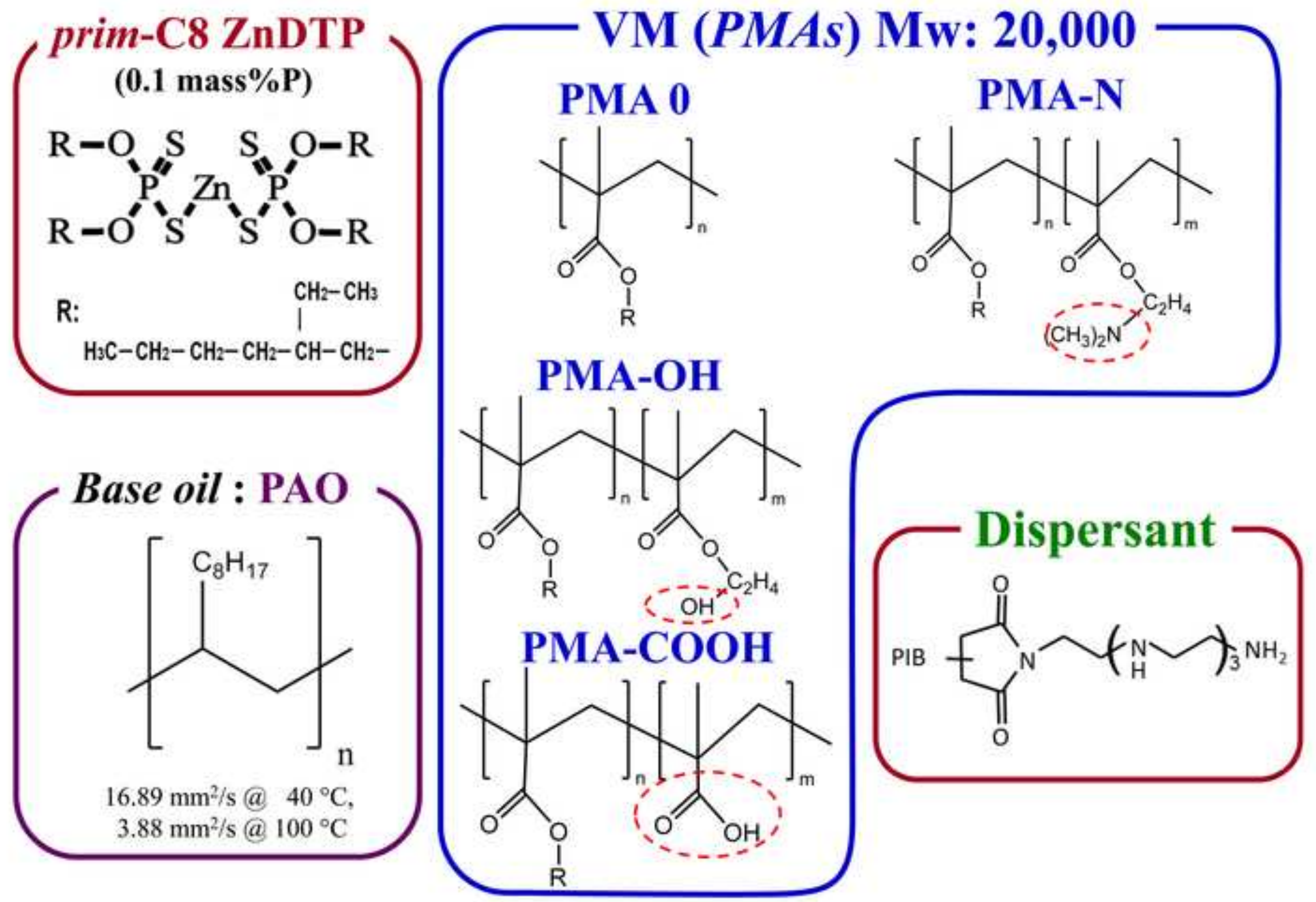




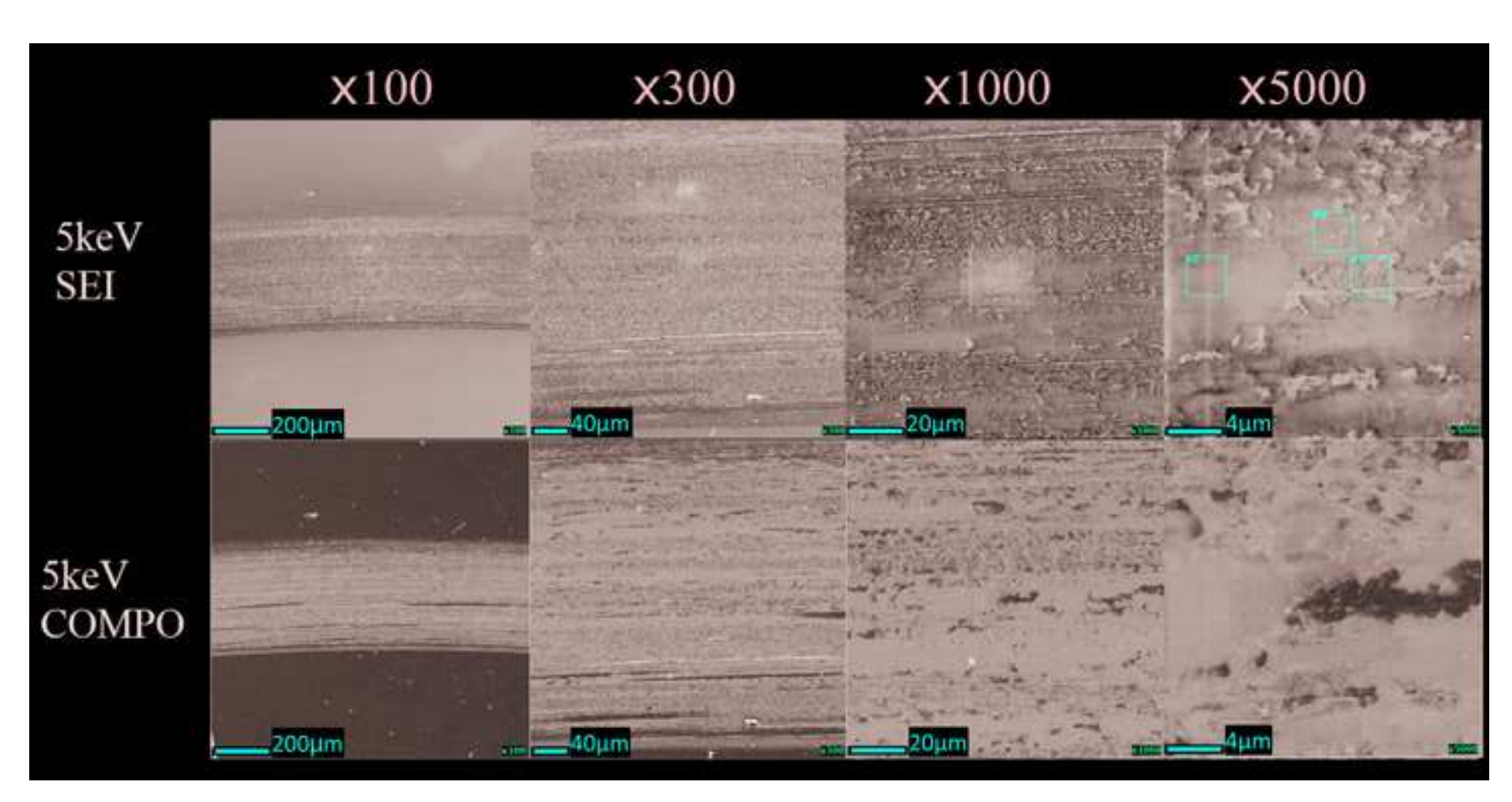

SEV

$5 \mathrm{keV}$ 

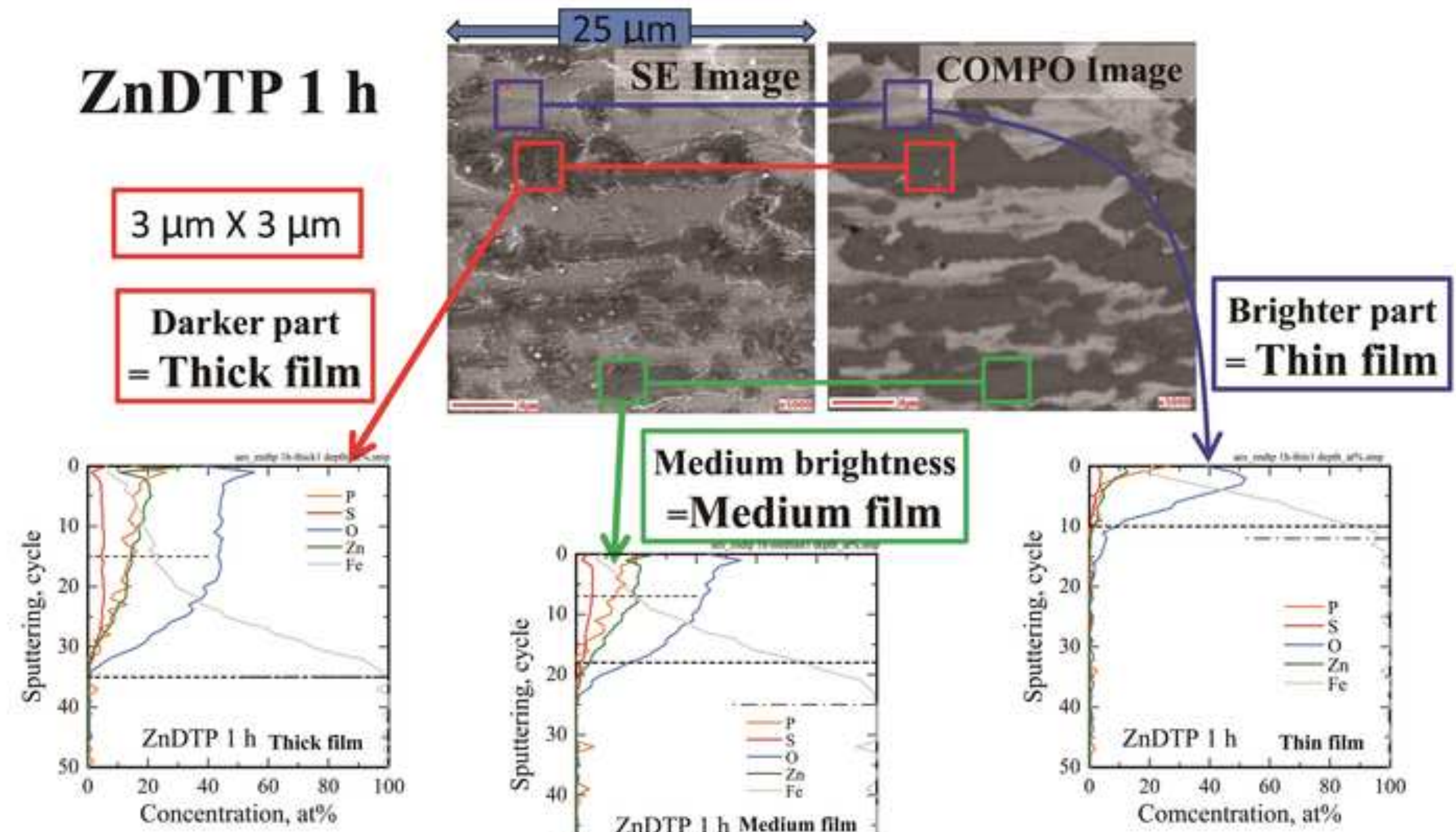
=Medium film

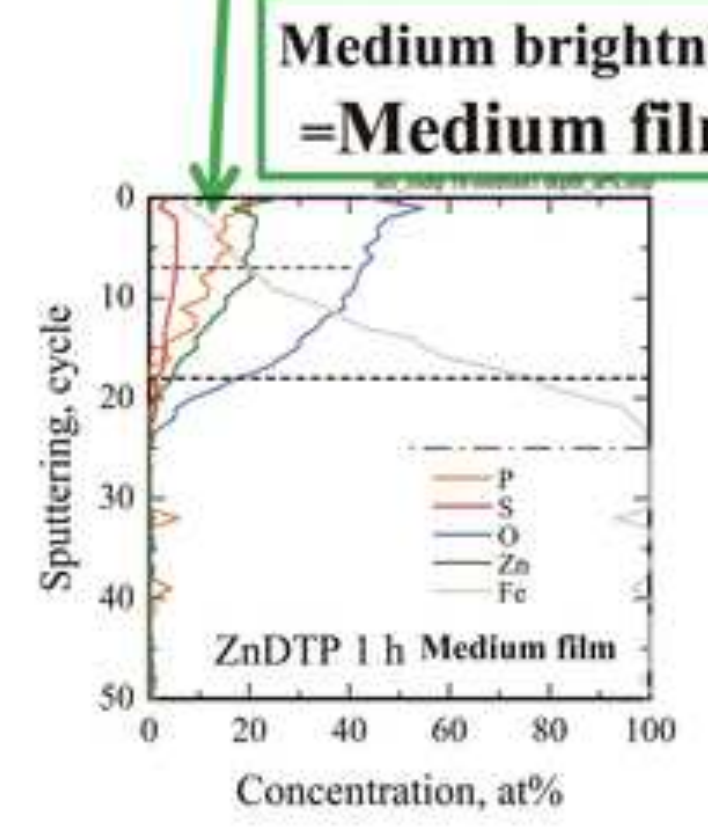

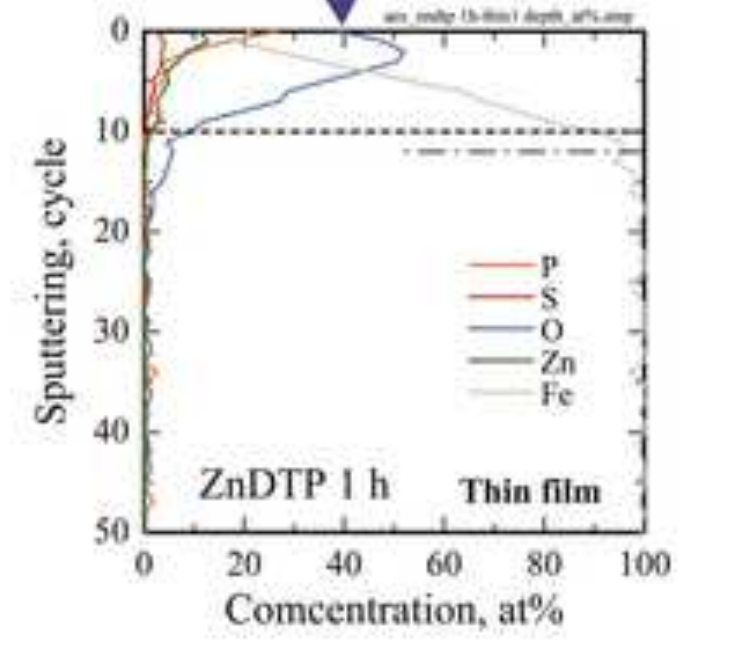




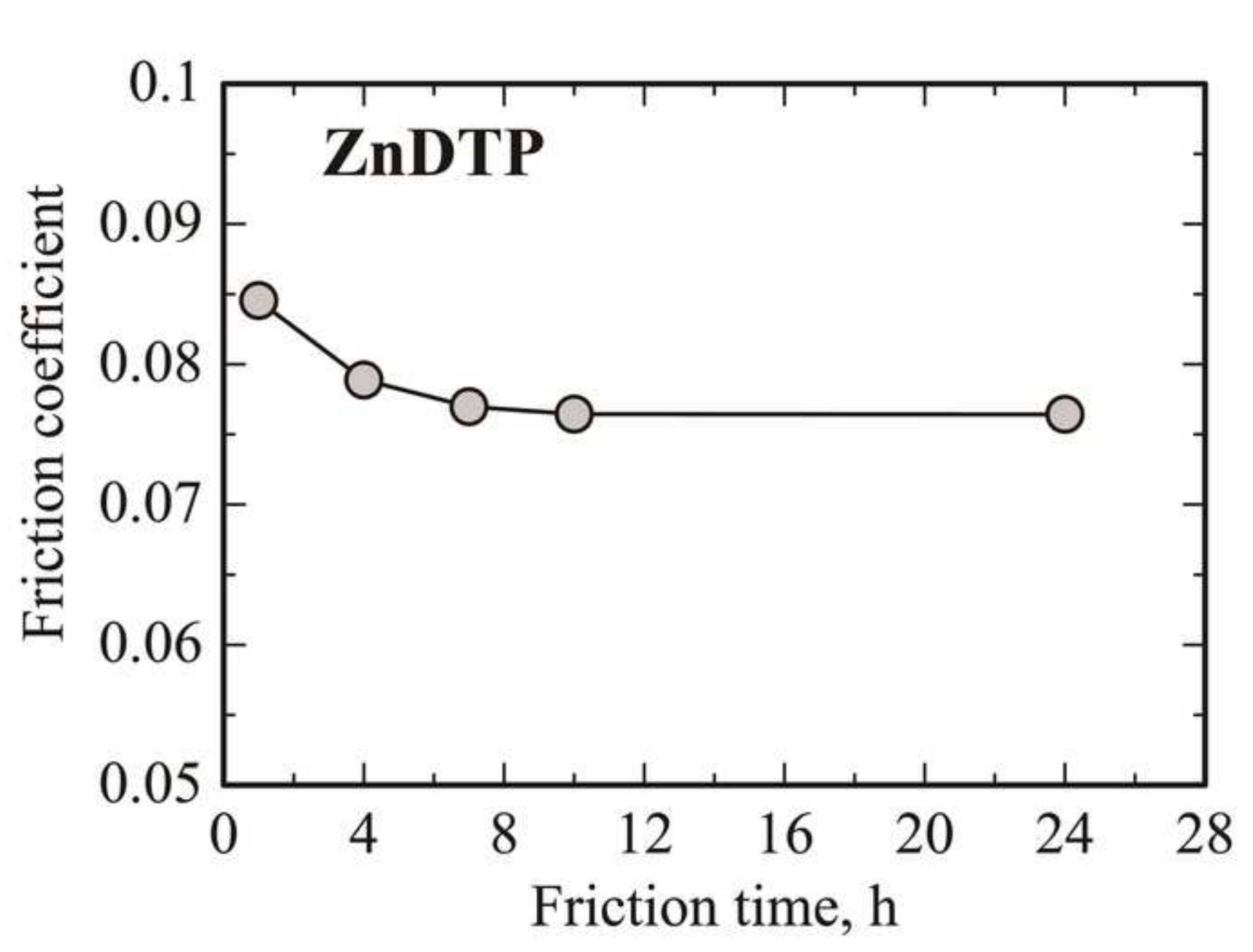



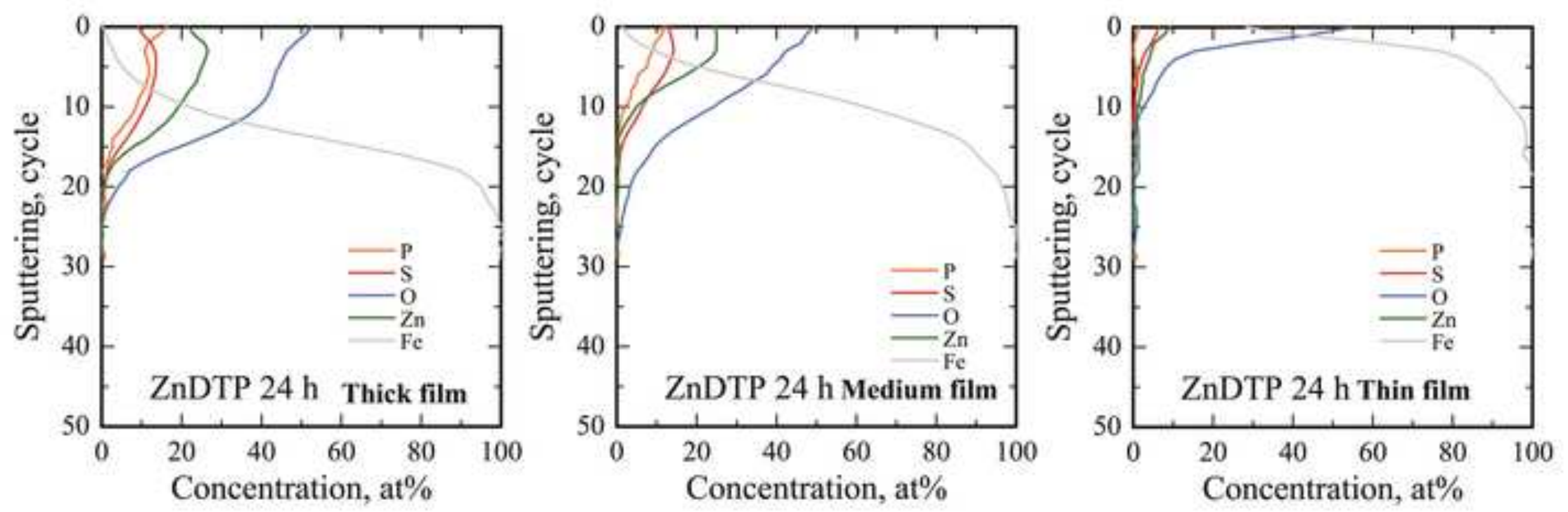


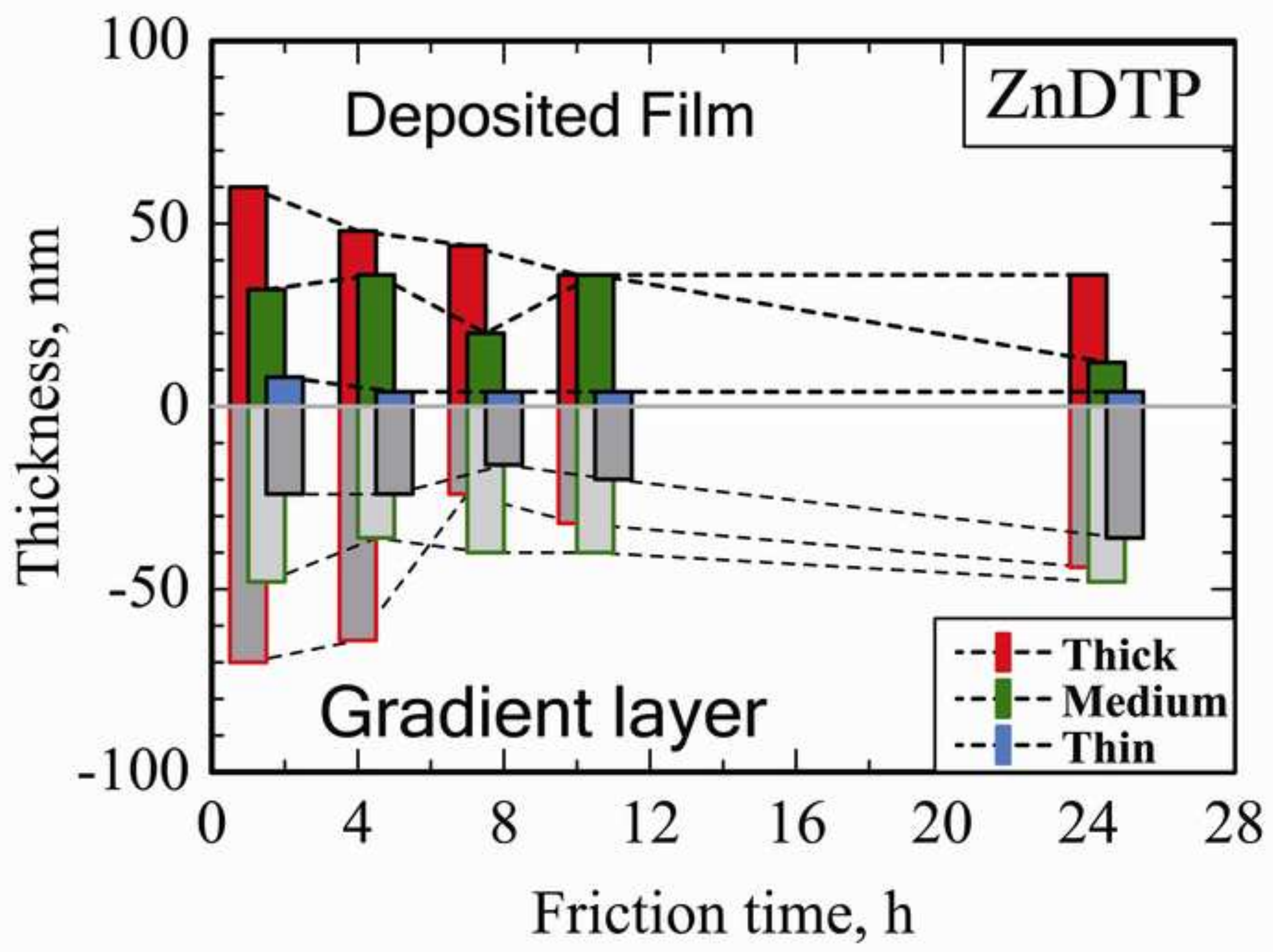




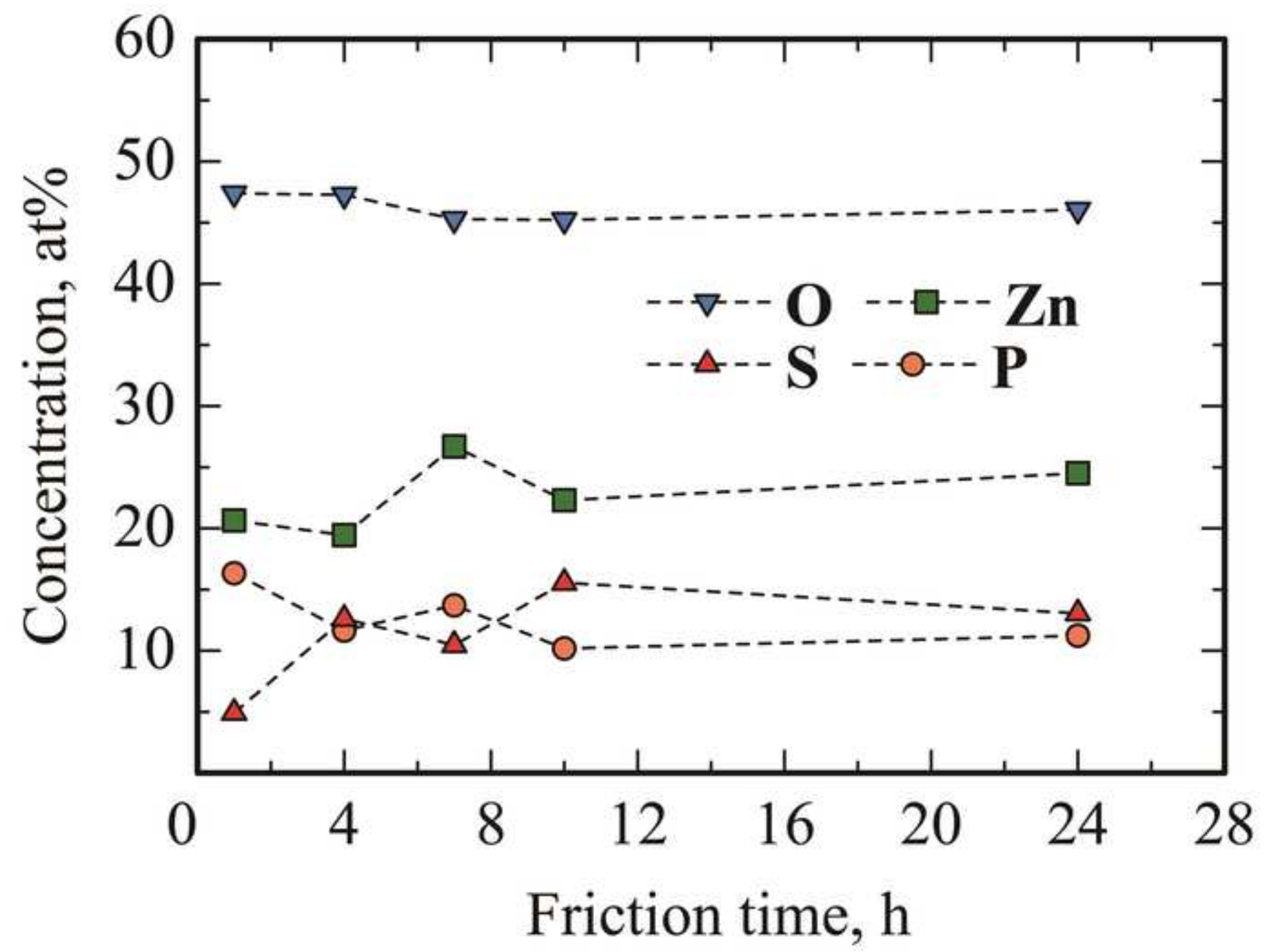




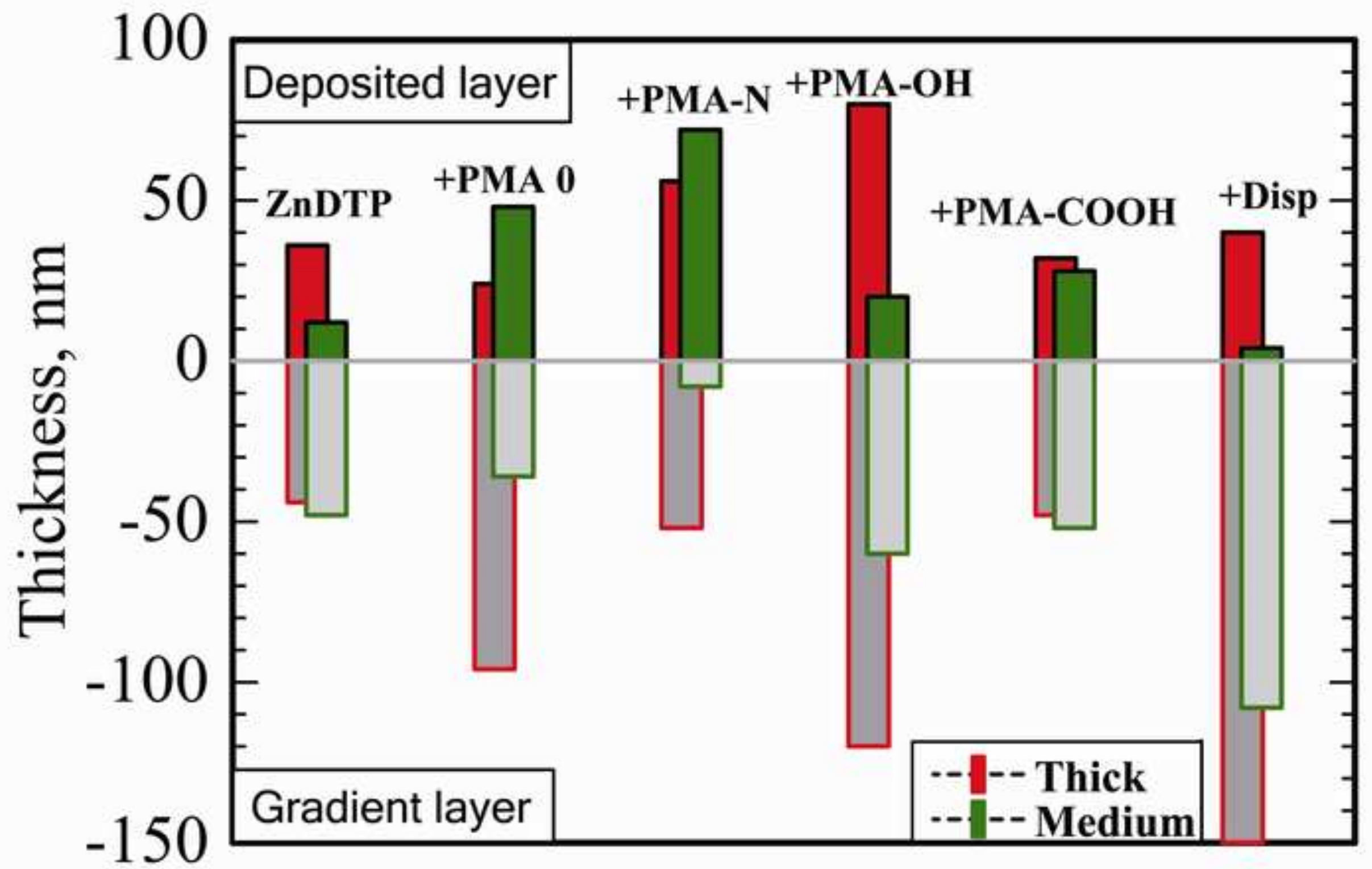




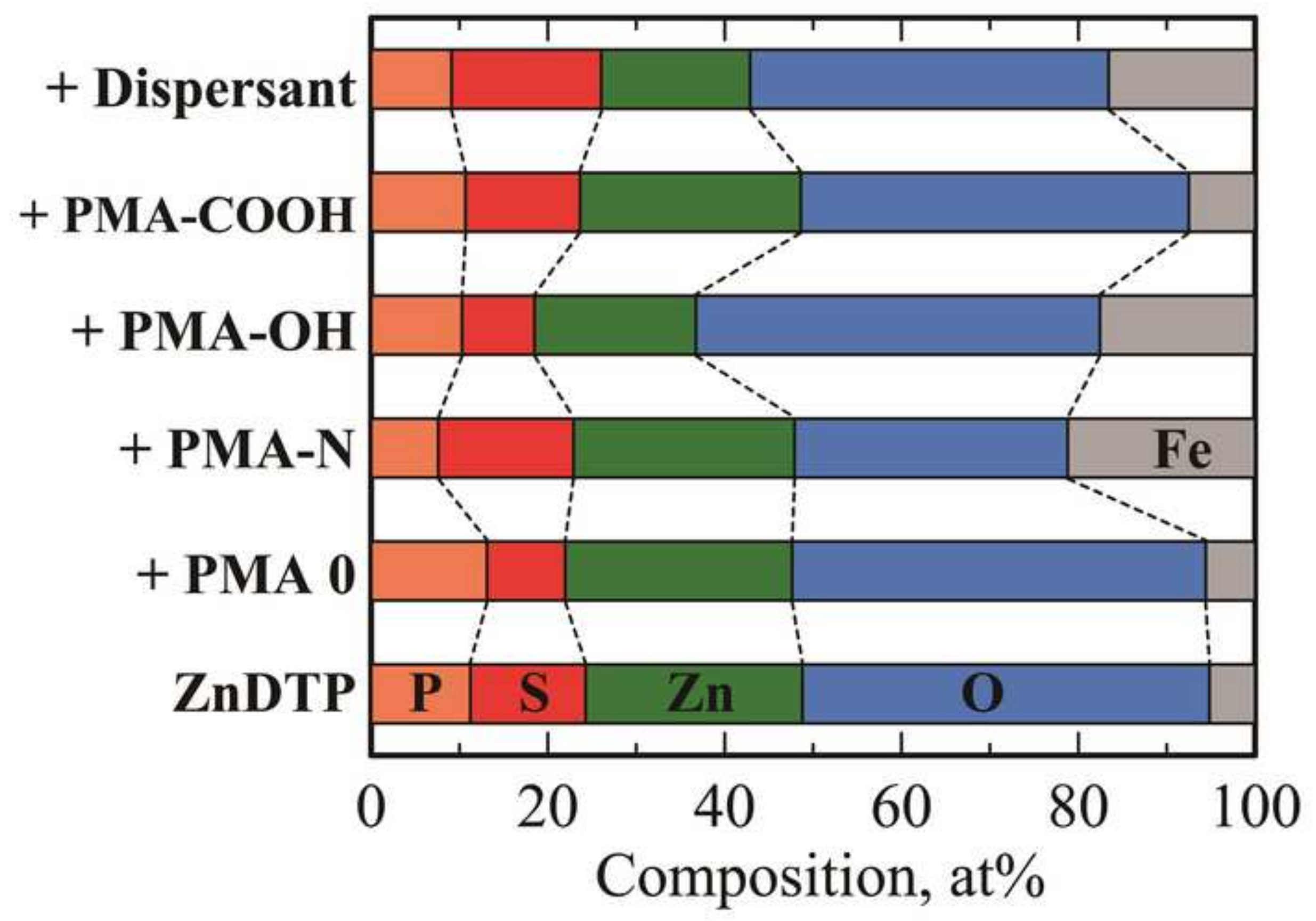



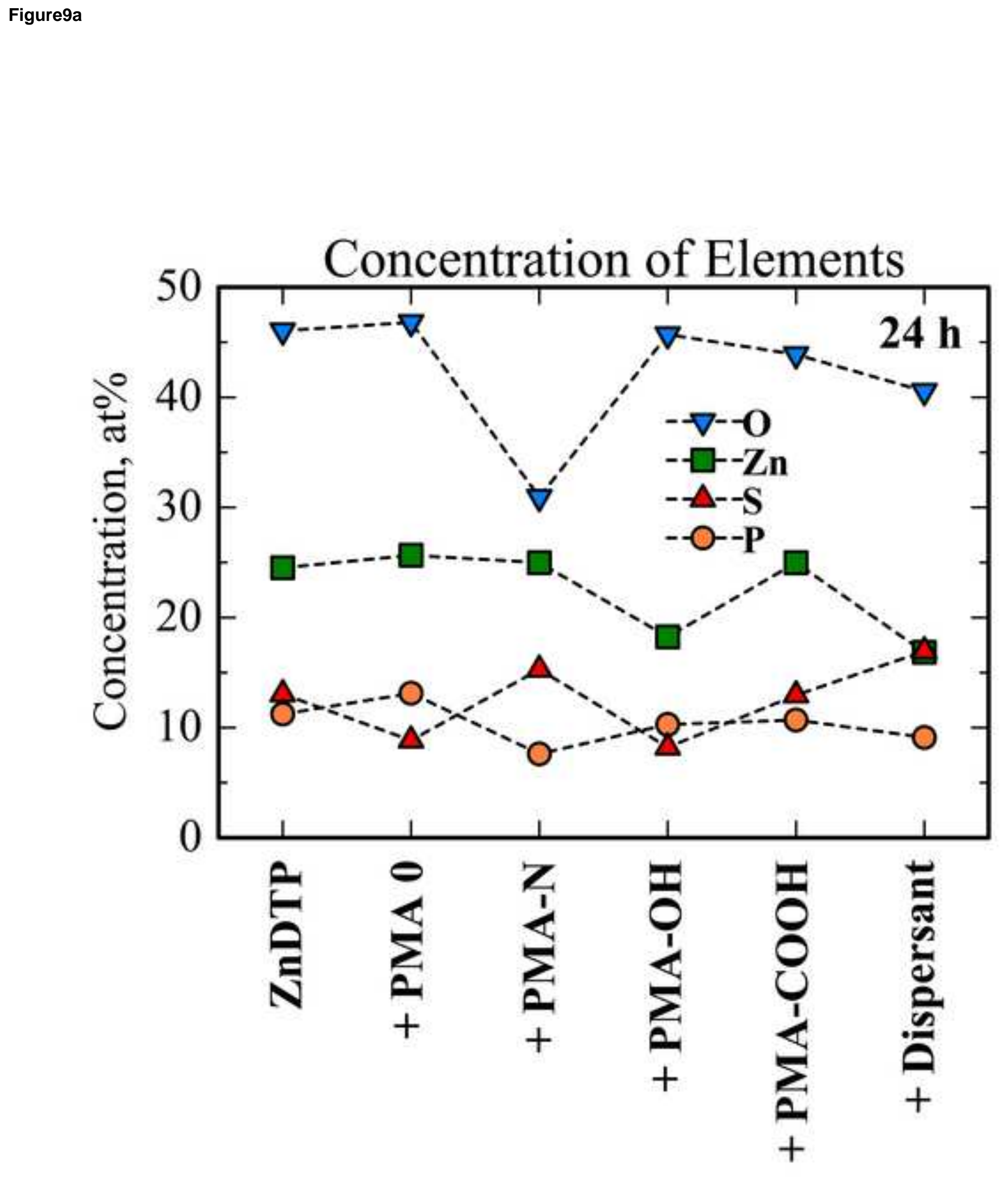

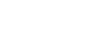

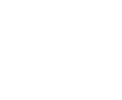

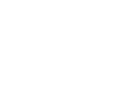

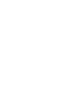

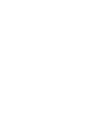

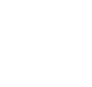

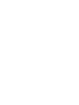

re

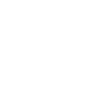


Relative Concentration

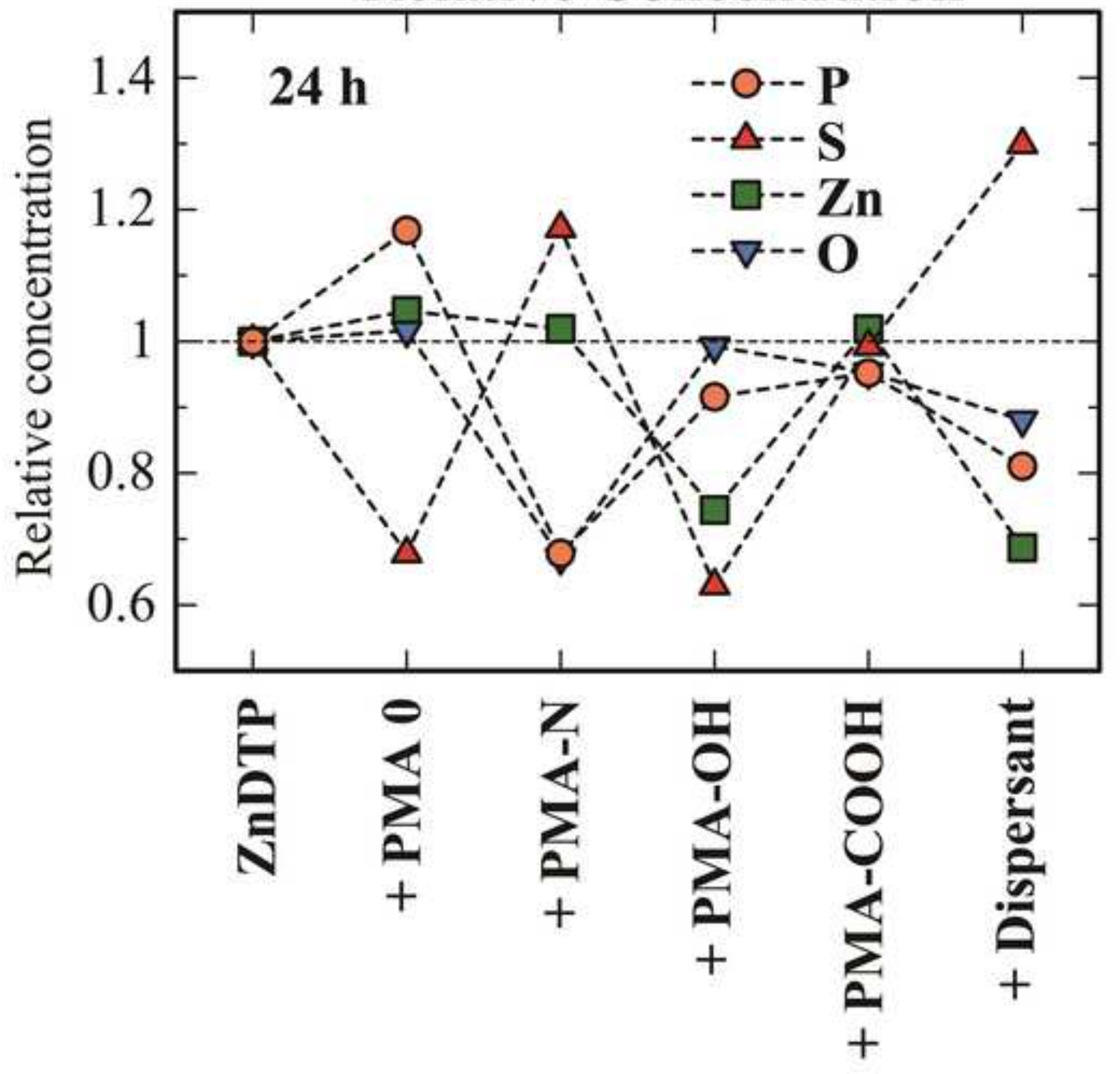




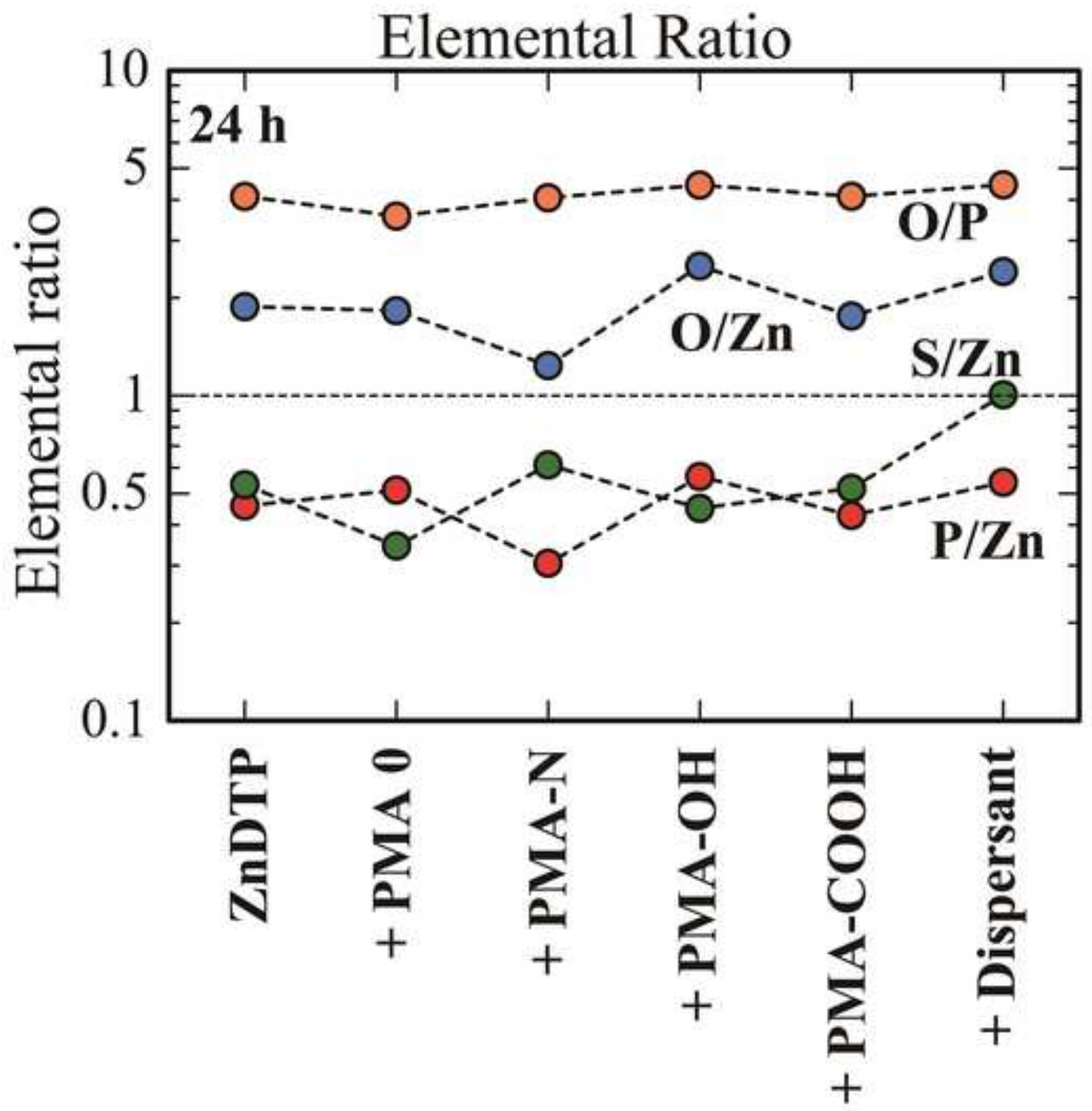




\section{ZnDTP}

Optical

Microscope
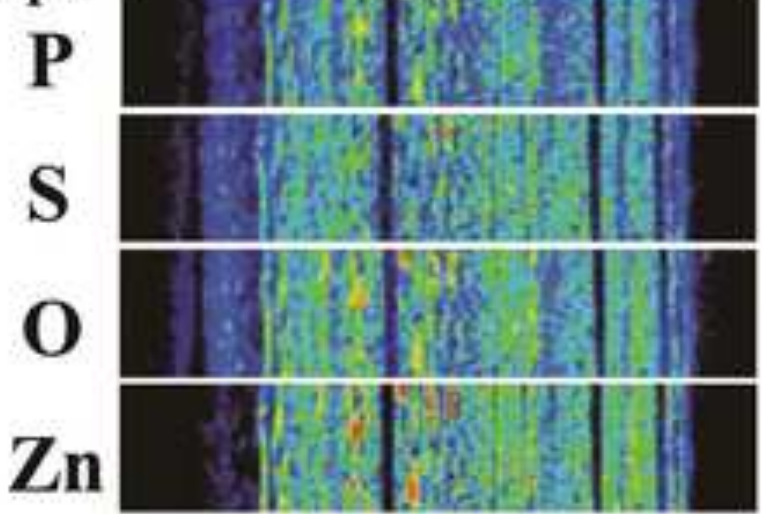

Optical

Microscope

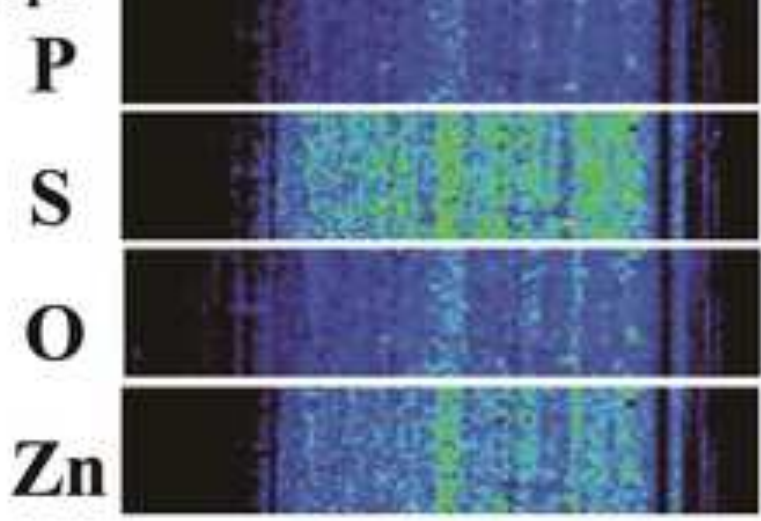

+ PMA-COOH

Optical

Microscope

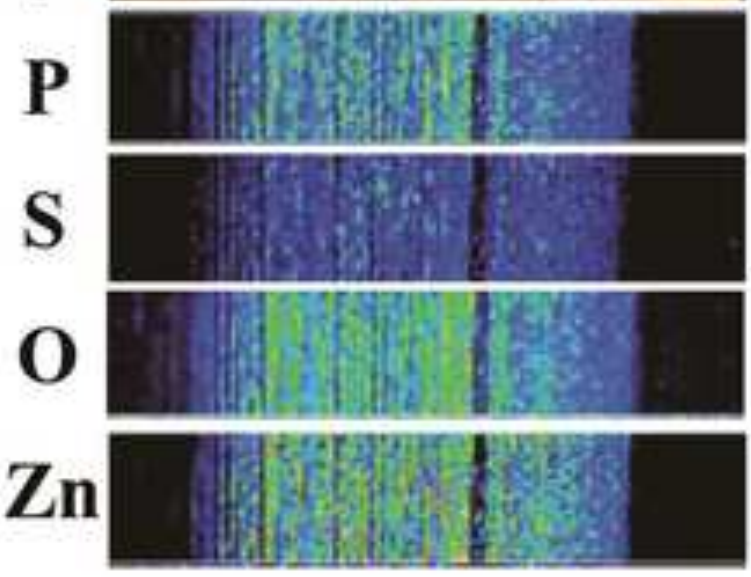

+ PMA 0
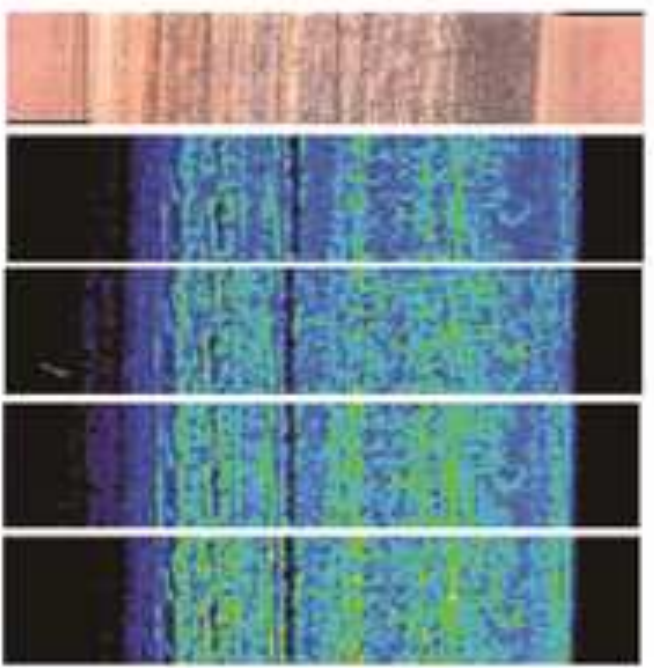

+ PMA-OH
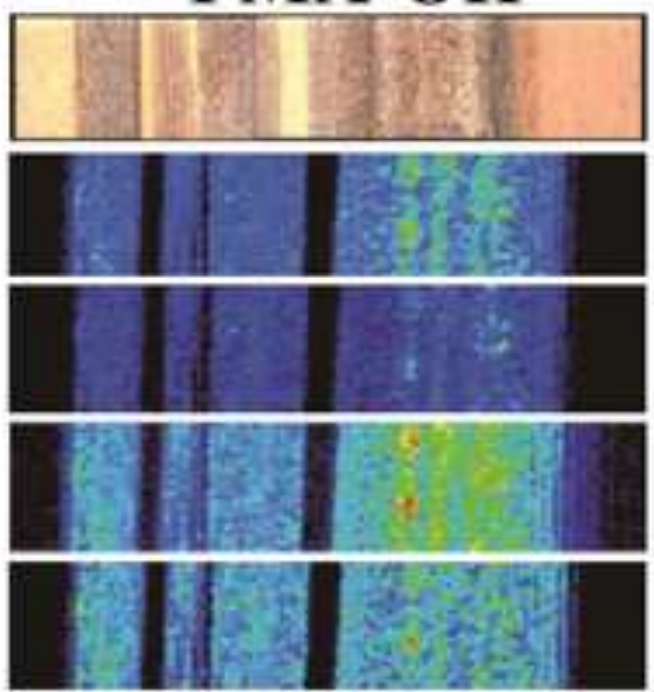

+ Dispersant
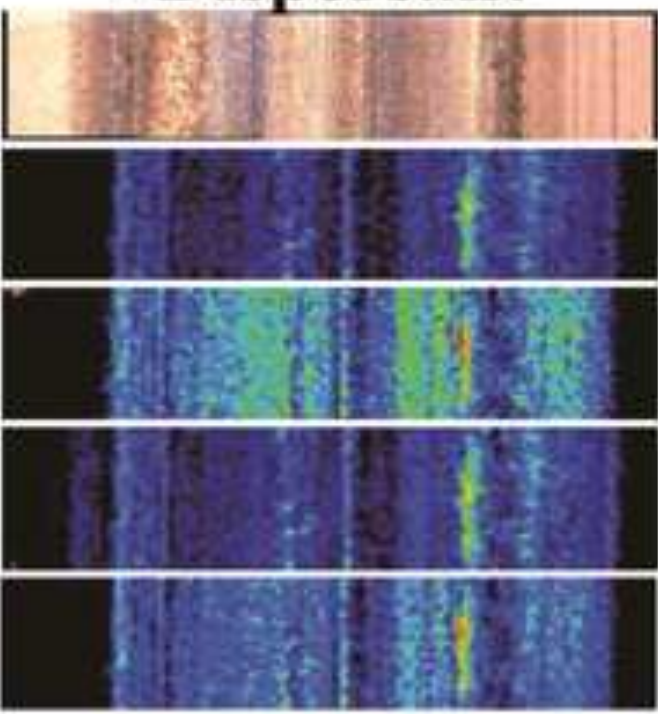

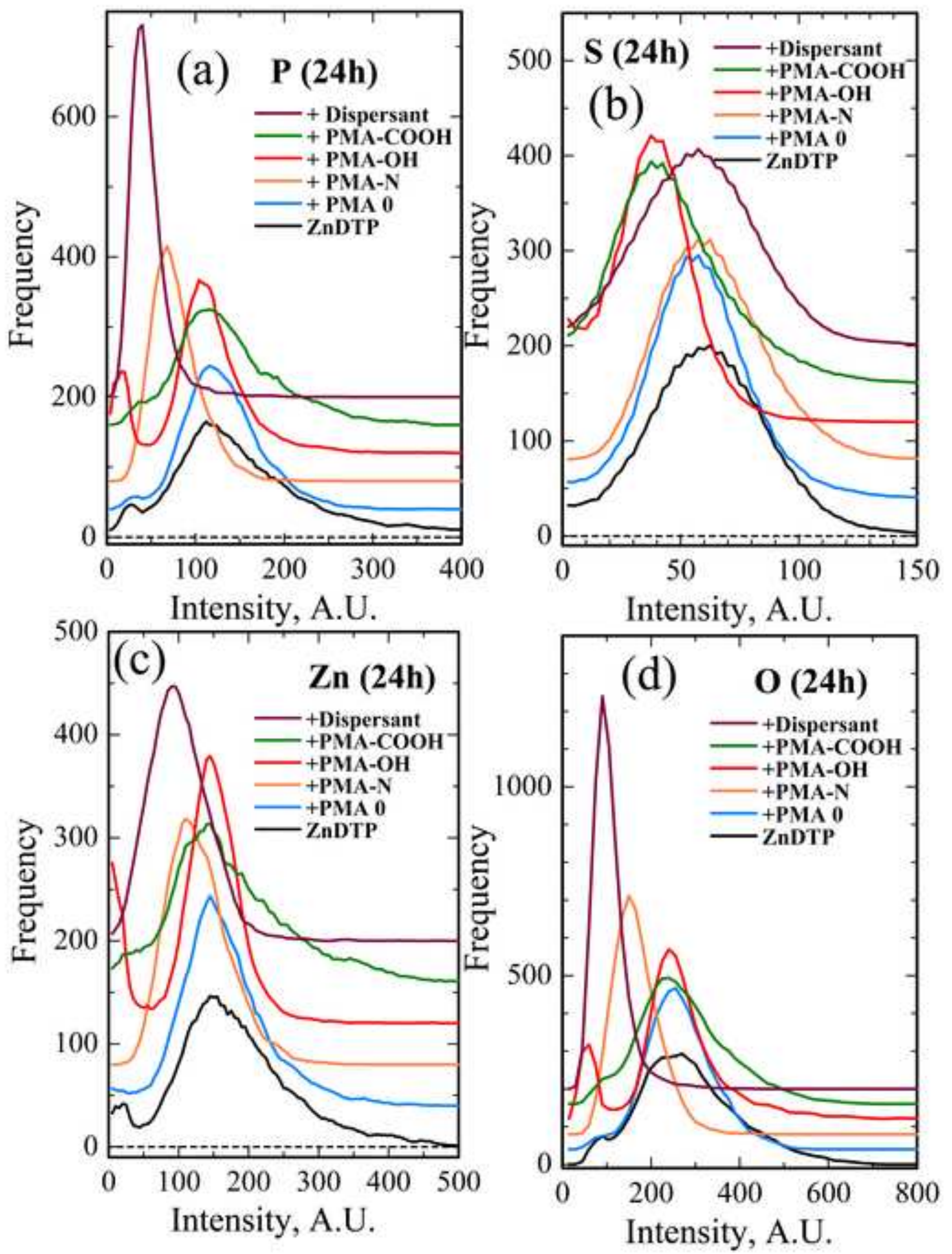

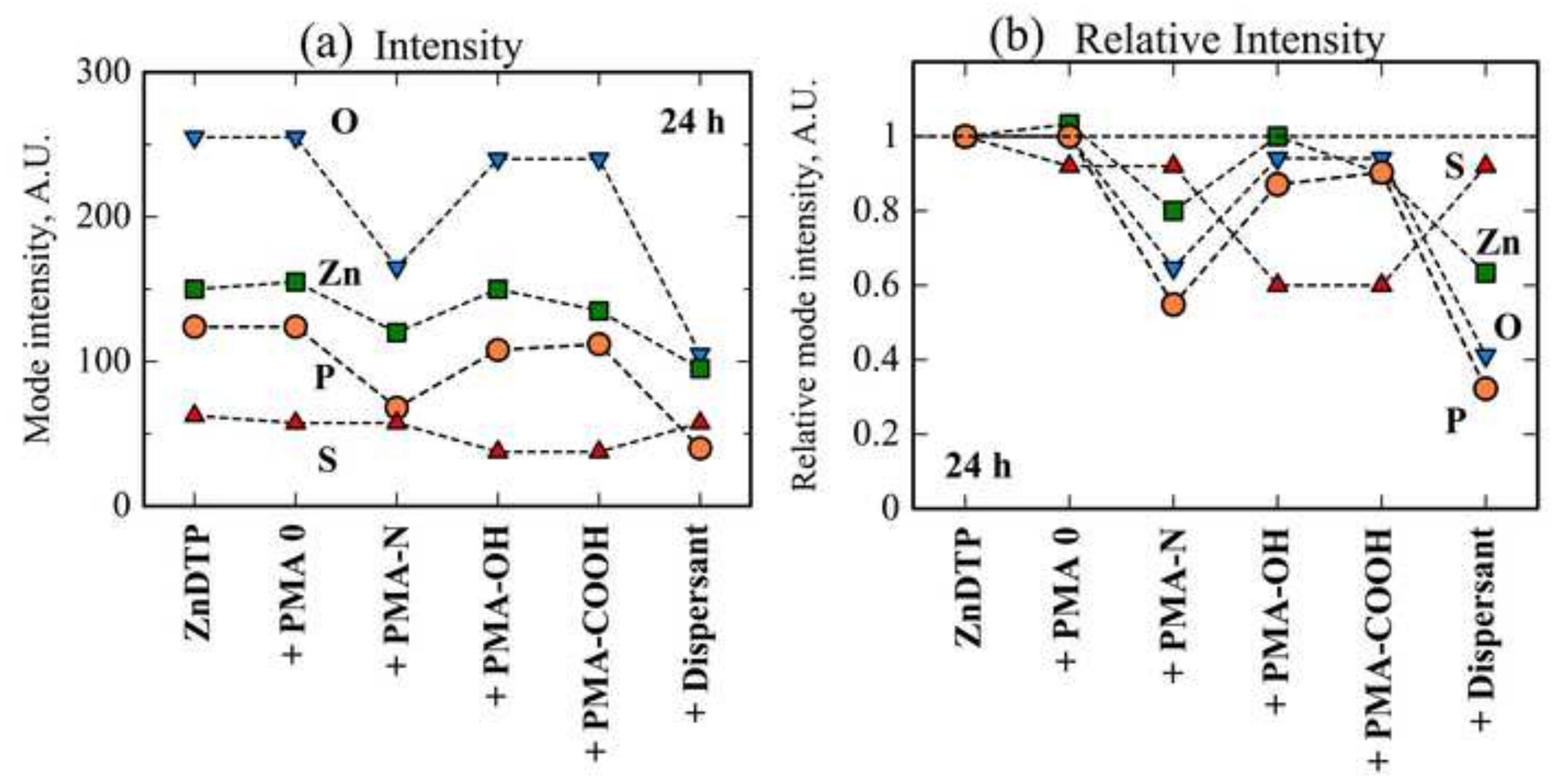

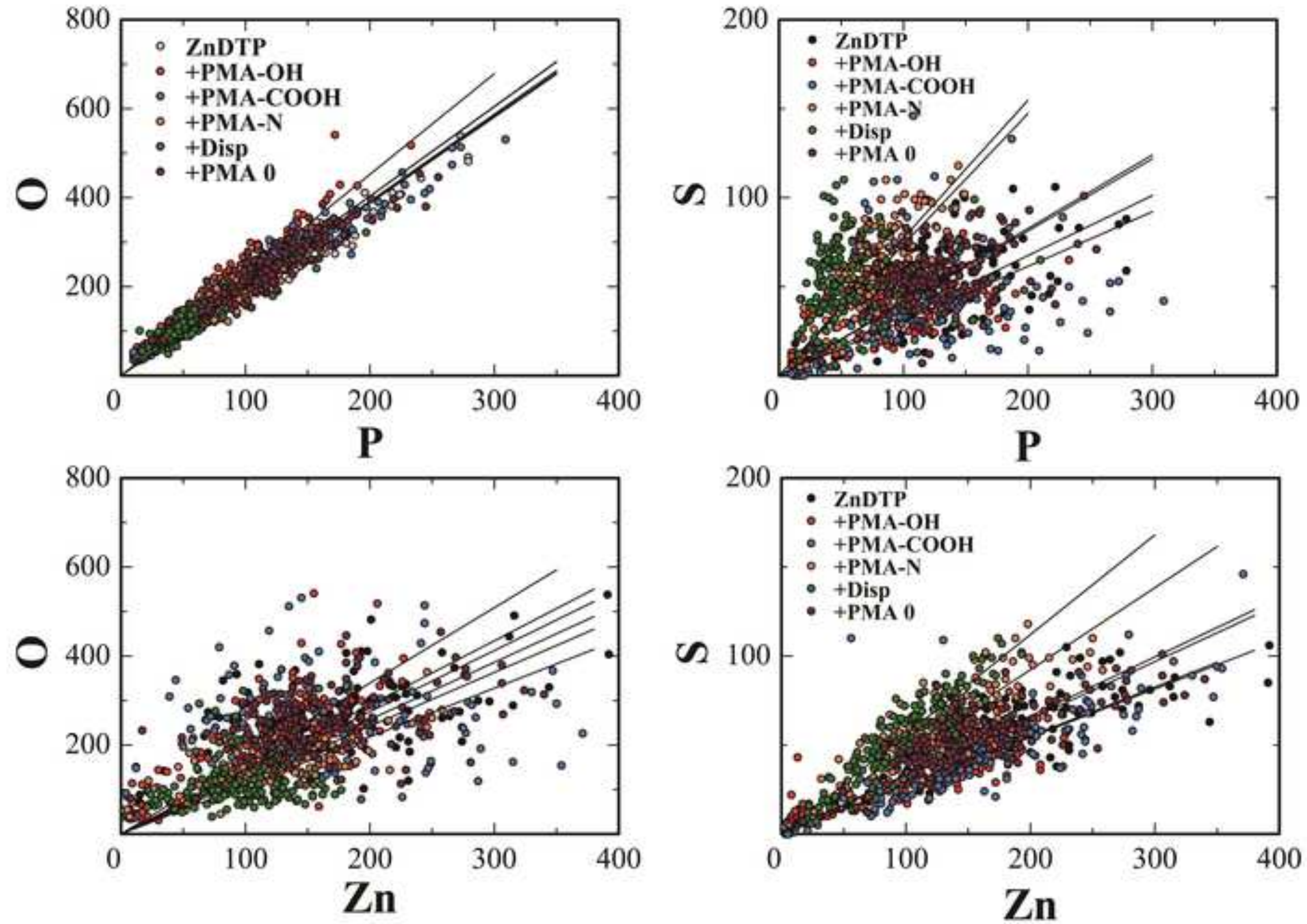


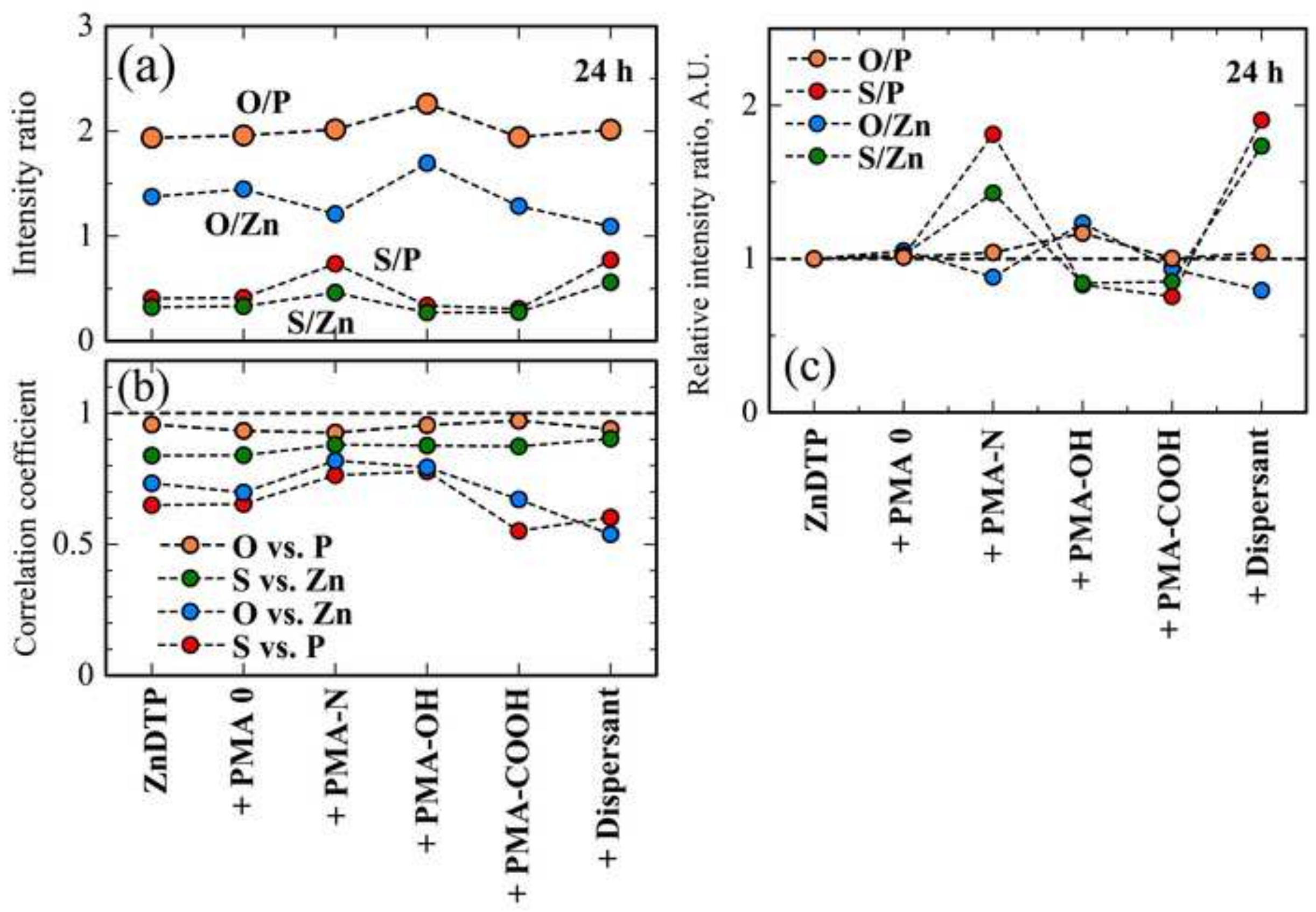




\begin{tabular}{|c|c|c|c|}
\hline & \multicolumn{3}{|c|}{ Experimental Condition } \\
\hline & Load & \multicolumn{2}{|c|}{$63.9 \mathrm{~N}\left(p_{\text {mean }}=667 \mathrm{MPa}\right)$} \\
\hline & Ball & \multicolumn{2}{|c|}{ SUJ2 (52100); 25.4 mm (dia.) } \\
\hline & \multirow{3}{*}{ Disk } & \multirow{3}{*}{ SK85 } & Inner dia. $16 \mathrm{~mm}$ \\
\hline & & & Outer dia. $27 \mathrm{~mm}$ \\
\hline & & & Thickness $3 \mathrm{~mm}$ \\
\hline & $\begin{array}{c}\text { Oil } \\
\text { Temperature }\end{array}$ & \multicolumn{2}{|r|}{$100^{\circ} \mathrm{C}$} \\
\hline $\begin{array}{l} \\
\vdots \\
\vdots\end{array}$ & $\begin{array}{c}\text { Entrainment } \\
\text { Speed }\end{array}$ & \multicolumn{2}{|r|}{$80 \mathrm{~mm} / \mathrm{s}$} \\
\hline & SRR & & $100 \%$ \\
\hline
\end{tabular}

